\title{
Intrinsic stability of quasicrystals under the generation of Frenkel pairs
}

\author{
J. Roth ${ }^{1}$, R. Schilling ${ }^{2}$, and H.-R. Trebin ${ }^{1}$ \\ ${ }^{1}$ Institut für Theoretische und Angewandte Physik, Universität Stuttgart, Pfaffenwaldring 57, W-7000 Stuttgart 80, \\ Federal Republic of Germany \\ ${ }^{2}$ Institut für Physik, Johannes-Gutenberg-Universität, Staudingerweg 7, W-6500 Mainz, Federal Republic of Germany \\ Received December 11, 1991
}

\begin{abstract}
Under irradiation metastable quasicrystals undergo a phase transition to an amorphous state. This transition can be reversed by annealing. As in normal crystalline materials the phase transition is considered to be triggered by generation and recombination of vacancies and interstitial atoms (Frenkel pairs). We have classified the possible Frenkel defects in a metastable monatomic quasicrystal with respect to geometric and energetic properties. With numerical simulation we have studied the behaviour of the quasicrystal under a load of Frenkel defects for various defect concentrations. We find three ranges of behaviour: up to $5 \%$ defects per atom the structure remains icosahedral, in a middle range it stays disordered icosahedral or it becomes either disordered or perfect crystalline, depending on the implementation of the defects. If there are more than $10 \%$ defects the structure becomes irreversibly amorphous. We finally compare our results with experimental data.
\end{abstract}

\section{Introduction}

Why quasicrystals are stable is an important, in general still unresolved question. Recently we have investigated this problem using perfect particularly decorated threedimensional Penrose patterns with atoms interacting via a Lennard-Jones-potential [1]. Similar work was performed in the following by Lançon and Billard [2].

Defects are important for the physical properties both of crystals as well as of quasicrystals. In particular, they influence the stability and can induce reversible or irreversible phase transitions.

Concerning the type of metastability of quasicrystals under the influence of point defects there are experimental and theoretical results available: in 1985 Urban et al. $[3,4]$ studied quasicrystals of the AlMn and AlV type with high voltage electron microscopy and showed they can be transformed in situ into an amorphous state when exposed to $500 \mathrm{keV}$ electron radiation. The transition is reversible: annealing of the irradiated sample reconstructs the quasicrystalline state. Bauer [5] extended the investigation to representatives of all known quasicrystalline classes and observed the transition for all of them.

A possible mechanism for the transformation has been proposed by Kronmüller et al. $[6,7]$ by a rate equation theory. Assuming that as in ordinary crystals the radiation defects are Frenkel pairs of vacancies and interstitials the shape of the phase boundary between the quasicrystalline and amorphous phase was deduced in an irradiation-temperature diagram. The phase transition is driven by generation and recombination of radiation defects.

It is the first intention of this paper to classify the types of interstitial sites of a specific monatomic threedimensional quasicrystal, the stable usp-structure, and to extend the definition of Frenkel defects to quasicrystals. Secondly, by computer simulations we investigate properties of these Frenkel defects, particularly the transformation to an amorphous state with increasing defect concentration. This is performed with a static gradient method. Finally we discuss the extension of the numerical simulations to the diatomic case shortly.

For the simulation method we refer to our previous article [1]. Only few details will be added in Sect. 2. Section 3 presents the classification of interstitials and Frenkel defects. In Sect. 4 we investigate the quasicrystal stability as a function of the defect concentration per atom $n_{d}$. Summary and conclusions are drawn in the final section.

\section{Generation, numerical relaxation, and analysis of the samples}

Details of the model used, its generation, the simulation method and the tools for analysis have been described in a previous paper [1], which we review briefly. The initial configuration always is a three-dimensional Am- 
mann-Kramer-Penrose lattice, which is constructed with the grid method. The lattice was decorated with one or two types of atoms, which were endowed with truncated Lennard-Jones-potentials. In particular we applied the unitsphere packing (usp) model, which results, if first atoms are placed to the vertices of the Ammann-KramerPenrose tiling. Subsequently one partner of each atomic pair connected by the short diagonal of the oblate rhombohedron is removed. Boundary effects are avoided by using rational approximants of the golden mean $\tau=\frac{1}{2}(\sqrt{5}+1)$ and periodic boundary conditions. With the help of the steepest descent method the initial configuration was relaxed.

The results were analysed by means of radial distribution functions (rdf), diagrams of the nearest neighbour bonds, bond orientation parameters [8] and by the binding energy of the quasicrystal which is defined as the negative of the potential energy per atom. The cell of periodic approximant with $p / q=5 / 3$ for $\tau$ contained 1902 atoms, which is equivalent to about 12 atoms along one period. Because of the relatively small dimension of the simulated volume, the structure factor, which is the Fourier transform of the rdf and which is normally measured in experiment, does not yield reliable information about the specific properties of a quasicrystal. It is not being considered in the analysis.

We checked the stability of the usp-model by displacing the atomic positions randomly until the system decayed to the amorphous state. The interval of mean displacement where the structure remains stable is comparable to that for a fcc-lattice.

For our investigations of defected monatomic quasicrystals we exclusively use the usp-decoration.

\section{Frenkel defects in quasicrystals}

One part of a Frenkel defect is a vacancy which as in periodic crystals is created by removing an atom of the ideal structure. The other part is a nearby interstitial. Instead of an immediate energetic characterization of interstitial sites we start with the Voronoi construction. Space is subdivided into Voronoi cells, which contain all points closest to a certain atom. For the fcc lattice the Voronoi cells, also known as Wigner-Seitz cells, are identical rhombic dodecahedra. The points in space most remote from the atoms (and therefore denoted "holes") are the corners of the Voronoi cells. The radius of a hole equals the distance of a corner to the central atom of the Voronoi cell and is the same for all adjacent atoms. Thus a hole carries a unique circumradius, which can be used for its classification in addition to the number of atoms. The centers of all Voronoi cells around a specific hole span another cell, the dual or Delauney cell. For the fcc-lattice the Delauney cells are tetrahedra and octahedra, forming the well-known interstitial types.

The concept of Voronoi decomposition is by no means limited to the case of lattices and is often used to describe liquid and amorphous structures. But then a large number of different Voronoi cell types occurs (see e.g. [9]). In quasicrystals, however, the number of Voronoi cell types is restricted (depending on the number of vertex neighbourhoods), and therefore we can distinguish interstitials as for ordinary lattices: by their circumradius, the number of neighbouring atoms and the shape of the Delauney cell, see below.

If the interstitial sites are filled with atoms, which are allowed to interact with their neighbours some of the interstitials become unstable. This dynamic feature modifying the geometric (kinetic) aspects will be discussed in Sect. 3.2.

\subsection{Classification of Frenkel defects for the primitive de- coration and the usp packing}

The interstitial sites of a perfect $3 \mathrm{~d}$ Penrose pattern having an atom at each vertex can be found by decomposing the prolate and oblate rhombohedra into Delauney cells. The prolate rhombohedron falls into two tetrahedra at the acute corners and an octahedron in between (Fig. 1a), similar to the primitive unit cell of the fccstructure. The oblate rhombohedron is composed of six
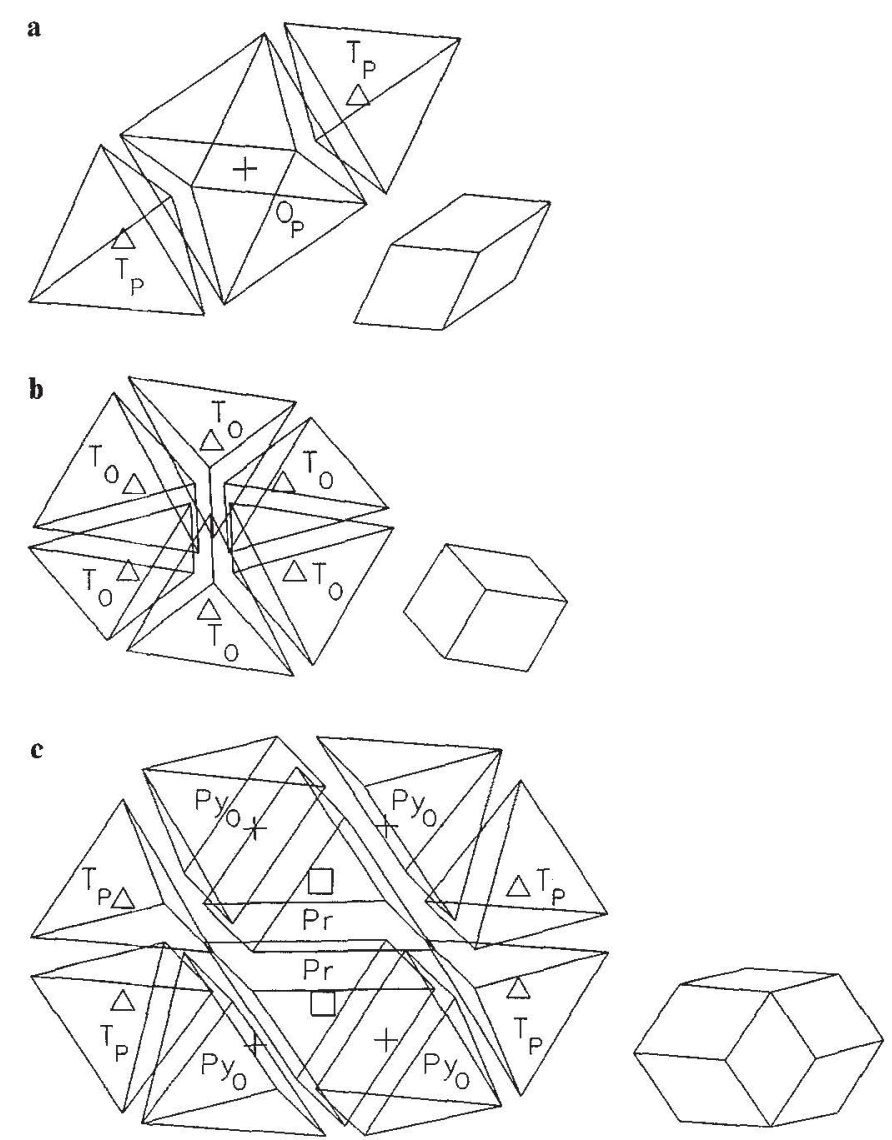

Fig. 1 a-c. Interstitial sites within the quasicrystal cells. a prolate rhombohedron, b oblate rhombohedron, $\mathbf{c}$ rhombic dodecahedron. The short hand notations are explained in the Tables 1 and 2 . $\triangle$ indicate tetrahedral interstitial sites in the prolate $\left(T_{p}\right)$ and in the oblate rhombohedron $\left(T_{o}\right),+\operatorname{octahedral}\left(O_{p}\right)$ and pyramidal $\left(P y_{0}\right)$, and $\square$ prismatic interstitial sites $(P r)$ 
Table 1. Interstitial sites in a three-dimensional perfect Penrose lattice. The quasilattice contains 2440 lattice points. The first column displays the type of the interstitial site and the second the cell of the quasilattice where it occurs. The radius is given in units of the edge length of both rhombohedra. The last two columns present the total number of interstitial sites and the number per quasilattice cell. The different types are shown in Fig. $1 \mathrm{a}$ and $\mathrm{b}$ and are indicated by their shorthand notation

\begin{tabular}{lllrl}
\hline $\begin{array}{l}\text { Type of } \\
\text { interstitial }\end{array}$ & Cell & Radius & Number & $\begin{array}{l}\text { Per } \\
\text { cell }\end{array}$ \\
\hline Tetrahedron $\left(T_{0}\right)$ & oblate rhombohedron & 0.596 & 5592 & 6 \\
Tetrahedron $\left(T_{p}\right)$ & prolate rhombohedron & 0.629 & 3016 & 2 \\
Octahedron $\left(O_{p}\right)$ & prolate rhombohedron & 0.726 & 1508 & 1 \\
Total number & & & 10116 & \\
\hline
\end{tabular}

tetrahedra of equal shape (Fig. $1 \mathrm{~b}$ ). The properties of the interstitials are listed in Table 1.

The characterization of the interstitials in the uspdecoration is more complicated. In the search for a decoration of the threedimensional Ammann-Kramer-Penrose pattern which allows a dense packing of spheres, Henley [10] proved, that all vertex separations are equal or longer than the rhombohedral edge length, except for those connected by the short diagonal of the oblate rhombohedron. Their distance is only about one half of the unit length. There are several methods proposed for the systematic elimination of one of those partner atoms in the literature [10-12]. We will discuss here the scheme of Henley because it specifies the cells in the so called usp-model.

The short bonds are not isolated but form wavy rings of ten atoms or parts thereof. Oblate rhombohedra are stacked along such a ring like pearls on a chain. Two neighbouring oblate and two additional prolate rhombohedra which have only one vertex in common form a rhombic dodecahedron (see Fig. 2). Now Henley removes every second atom of the rings, which is equivalent to taking away the only atom inside the dodecahedron. All the remaining atoms have a separation of about unity. We are left with a structure without oblate rhombohedra, only containing prolate rhombohedra, rhombic dodecahedra and more complicated cells resulting from the fragmentation of the rings. The usp-model cannot be decomposed merely into simple rhombohedral cells.

To find the interstitial sites we have to decompose the prolate rhombohedron as performed in Fig. 1 a, the rhombic dodecahedron and the complicated cells into Delauney cells.

The dodecahedron divides into four tetrahedral cells of the type found in the prolate rhombohedron, four pyramidal cells which have the same radius as the octa-

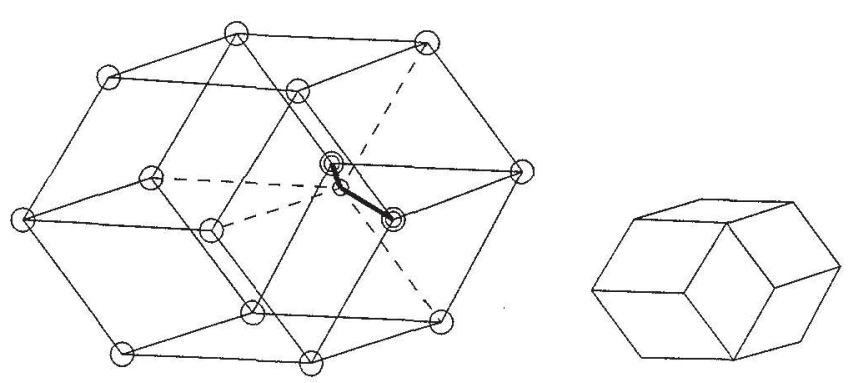

Fig. 2. Rhombic dodecahedron. The dodecahedron, indicated by thin lines, is build up by two prolate (PR) and two oblate rhombohedra (OR). At the left side are the PR placed on top of each other and at the right side the OR behind each other. The edges lying inside the dodecahedron are marked by dashed lines. In the primitive decoration all vertices (circles) are occupied by atoms. The thick lines mark the short diagonals of the OR which are part of a ten-member ring. Applying the usp-construction amounts to removing the atom marked by the single small circle, whereas the atoms with the double circles remain. The resulting cell is an empty dodecahedron
Table 2. The interstitials in the usp-decoration. The quasilattice contains 1902 lattice points. The horizontal lines divide the table into blocks of interstitial sites with the same radius. The first row of a block gives the original site in case of the octahedral and prismatic interstitial sites, the others are obtained by removing some corners (derived). The last block contains the interstitial sites in the complicated cells. They are grouped into two types due to the energy of the test particles placed there. The first and second column indicate the type of the interstitial site and the cell, respectively, if known, in the usp-decoration. The third column displays the radius of the interstitial site in units of the edge length of both rhombohedra. The last two columns show the total number of interstitial sites and the number of stable ones. The defects are drawn in Fig. 1 a and $\mathrm{c}$ and are indicated by their shorthand notation

\begin{tabular}{lllll}
\hline $\begin{array}{l}\text { Type of } \\
\text { interstitial sites }\end{array}$ & Quasilattice cell & Radius & $\begin{array}{l}\text { Total } \\
\text { number }\end{array}$ & $\begin{array}{l}\text { Number of } \\
\text { stable ones }\end{array}$ \\
\hline Tetrahedron $\left(T_{p}\right)$ & $\begin{array}{l}\text { Prolate rhombohedron } \\
\text { Rhombic dodecahedron }\end{array}$ & 0.629 & 2770 & 2770 \\
Octahedron $\left(O_{p}\right)$ & $\begin{array}{l}\text { Prolate rhombohedron } \\
\text { Rhombic dodecahedron }\end{array}$ & 0.726 & 533 & 533 \\
Pyramid $\left(P y_{0}\right)$ & 0.726 & 1199 & \\
Tetrahedron $\left(T_{o}\right)$ & Rhombic dodecahedron & 0.726 & 632 & \\
Prism $(P r)$ & 0.789 & 548 & 548 \\
Pyramid $\left(P y_{P r}\right)$ & & 0.789 & 328 & \\
Tetrahedron $\left(T_{P r}\right)$ & & $0.814-0.820$ & 283 & 283 \\
Further types: & & $0.845-0.911$ & 251 & 72 \\
medium level & & & 7151 & 4206 \\
low level & & & & \\
\hline Total & & & & \\
\hline
\end{tabular}


hedral cells in the prolate rhombohedron, and two prismatic cells of Fig. $1 \mathrm{c}$.

The holes of the more complicated cells are found and classified by a computer program. As in general they are difficult to describe and of low frequency, we did not study them in detail.

The Delauney cells, i.e. the types of interstitial sites are summarized in Table 2. The table is divided into four horizontal blocks, where in each block the holes with same radius but with different shape are collected. The cell with the largest number of corners is shown at the top of each block. The complicated cells are also given.

\subsection{Stability of the interstitial sites}

By decomposing space into Voronoi cells (Sect. 3.1), we found 16 different geometric types of intestitial sites, with 11 different radii. The geometric types can be distinguished by their shape or by the energy of a test particle. The geometric classification is based on the assumption, that the Lennard-Jones potential energy, which a test atom experiences in the field of all other atoms, displays a minimum at or close to the center of the Delauney cell. But this is not the case in general. Some of the Voronoi cell corners are degenerate: in this case the vertex lies on a face or an edge of the corresponding Delauney cell. If one places a test particle at such a hole and leaves it free to move under the forces of the neighbouring atoms it will fall into the center of an adjacent Delauney cell. Therefore the hole is said to be unstable. But even if it rests in the interior of the cell it can be unstable because of the low symmetry or irregular shape of the cell.

We have examined the stability of all holes with test particles. The ordinary lattice atoms are kept fixed at their place, the test particle is placed at the geometric interstitial site, and is then released. Fixing the atoms is necessary because if the lattice atoms also relax, then the entire structure of interstitials would change locally, complicate the simulation and spoil the classification scheme.

As described above there exist some geometric types with identical radius but different number of corners of the Delauney cell. We denote the cell with the highest number of corners or neighbouring atoms the original one and the others the derived ones. These are obtained from the former ones simply by removing some corners (Fig. 3). We find in general that the original holes are stable and the derived ones are unstable.

Figure 4 demonstrates this fact for a pyramid lying on a prism. The pyramid has the same shape as the octahedron of radius 0.726 , but one corner is missing. It is unstable. The test particle moves to the larger prismatic hole $(r=0.789)$.

After testing the stability we can classify the holes into 8 types using the energy of the relaxed test particles. The total number of stable holes is much less than the number of geometric ones because different starting sites often lead to the same final place. Most of the remaining
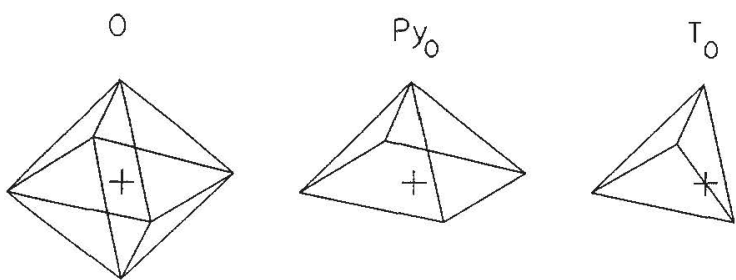

Fig. 3. Unstable cells derived from the octahedral interstitial sites. The diagram displays a stable octahedral cell out of the prolate rhombohedron, an unstable pyramidal cell where a corner has been removed with respect to the first cell, and a tetrahedral cell with two corners cut off. The pyramidal cell is part of the rhombic dodecahedron (see Fig. 1). All cells have the radius of the octahedral cell in Fig. 1
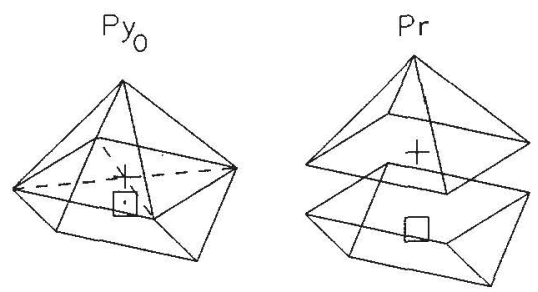

Fig. 4. Interstitials in the quasicrystal cells. The diagram displays a cluster of a pyramidal and a prismatic cell which is part of the rhombic dodecahedron. The pyramidal hole lies on the basis and on the face of the prism. Its radius is 0.726 compared to 0.789 of the prismatic hole. Both radii are less than the nearest neighbour distance and therefore the force of the neighbouring atoms is repulsive. The interstitial is moved to the larger prismatic hole because no potential barrier exists due to the degeneration of the pyramidal interstitial site. The symbols are the same as in Fig. 1

holes can be identified with corresponding stable geometric holes (see Table 2 and Fig. 5). The solid line in Fig. 5 indicates the energy change of the transition in Fig. 4.

Several schemes were applied to the generation of Frenkel pairs:

1. Uncorrelated geometric defects (ugd). A certain number of vacancies was generated randomly and their corresponding atoms were distributed randomly over the stable and unstable holes sites. Only octahedral holes were examined in this scheme.

2. Correlated geometric defects (cgd). Again atoms were removed randomly but were placed into holes belonging to the second shell around the vacancy. (The first shell belongs to the Voronoi cell of the vacancy and is not stable.) Here we studied tetrahedral, octahedral and prismatic holes and a mixture of all types of interstitials.

3. Uncorrelated physical defects (upd). They were generated as in the first scheme, but we used stable holes only and investigated separately tetraheral, octahedral, and prismatic interstitials, and in the complicated cells stable holes of medium energy level as well as of low energy level.

As we did not find any significant difference between correlated and uncorrelated geometric defects we did not further investigate correlated physical defects (cpd). 


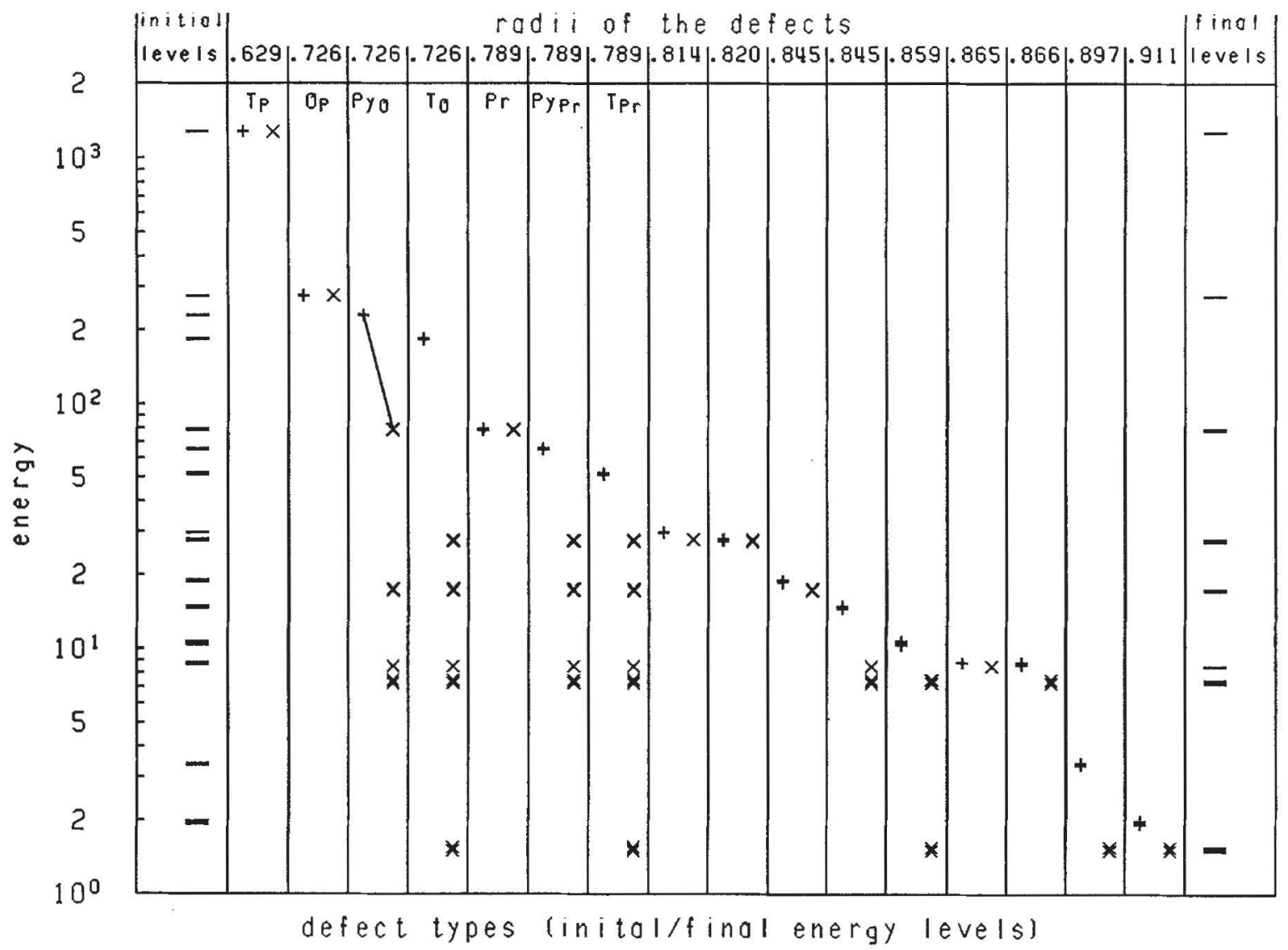

Fig. 5. The relation between geometric and physical defects. The first column of the diagram displays the initial energy level of the test particles placed at the corners of the Voronoi cells (geometric defects). The other columns contain for each type of interstitial sites at the left side the initial energy $(+)$ for a test particle and at the right the final energy $(x)$ after relaxation. There may be different final states due to the fact that there are different neighbouring cells. The interstitial sites are characterized by their radius as well as by their shorthand notation (see Table 2). The last column displays the collection of all possible final energy levels. Only eight types of defects are left. They can be related to the stable geometric types by inspection of the diagram. The solid line in the fourth column indicates the transition of an unstable pyramidal interstitial to a stable prismatic one (sixth column) as is explained in Fig. 4

Apart from the systematic investigation of one realization of interstitial sites for all interstitial types we studied a series of realizations of the octahedral ( 5 altogether) and prismatic sites ( 2 altogether) to improve the statistical significance of our results. For the same reason we also produced ten additional realizations of mixed $\mathrm{cgd}$ defects with a concentration of $1.5 \%$ and twenty realizations of octahedral cgd and upd defects with a concentration of $7.5 \%$. The octahedral defects where chosen because of their large number and radius, and because of their shape which is similar to that of the octahedral interstitials in the fcc lattice.

\section{Results}

The defect configurations generated as described in Sect. 3.2 were analysed by several tools:

1. By determining $E_{b}$, the binding energy per atom, which is the negative of the potential energy per atom and is a measure of stability.
2. By the radial distribution function $G(r)$ characterizing the translational order.

3. By bond-diagrams displaying the orientational order. The directions of the nearest neighbour bonds are projected onto an octant of a sphere spanned by pairwise orthogonal twofold symmetry axes of the icosahedron. The density of bond directions can be characterized by the bond order parameters $Q_{l}$ of $[1,8]$.

With respect to these quantities we can divide the range of defect concentrations $n_{d}$ into three different sectors with five different structures as demonstrated in Figs. 6 and 8 .

Figure 6 presents the evolution of $E_{b}$ during relaxation for three typical values of $n_{d}$ representative for a finite range of defect concentration. In addition to $E_{b}$ we present the binding energy per atom $E_{b}^{\mathrm{fcc}}$ of an ideal fcc lattice (fcc.id.). The initial defective configuration relaxes either to an icosahedral $\left(n_{d}=1.5 \%\right)$, fcc $\left(n_{d}=7.5 \%\right)$ or an amorphous structure $\left(n_{d}=20 \%\right)$. In case of the fcc structure, $E_{b}$ is less than $E_{b}^{\mathrm{fcc}} \approx 3.26$. This discrepancy comes from vacancies in the relaxed fcc lattice. Because 

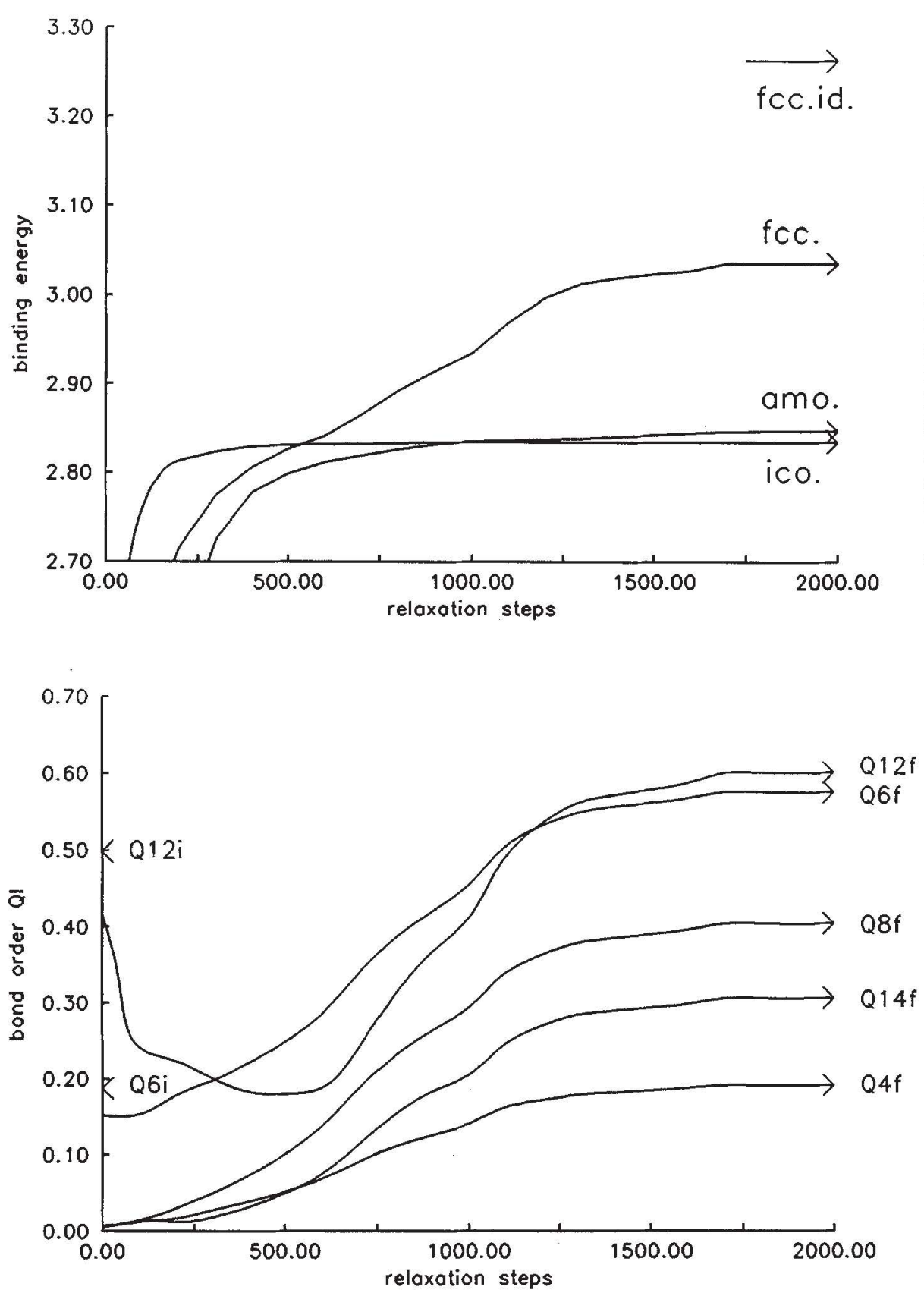

Fig. 6. The evolution of the binding energy during relaxation. Three examples representative for the three ranges of behaviour are given. The initial structures only differ by the concentration of defects: the final icosahedral, fcc- and amorphous structure corresponds to $2.5 \%, 7.5 \%$ and $20 \%$ of defects per atom. The final binding energy for the cubic crystal, the icosahedral usp-decoration and a typical amorphous sample are represented by arrows. The energy level of a perfect fcclattice without holes is indicated by fec.id. The difference between fec and fcc.id. is explained in the text
Fig. 7. The evolution of the bond order parameter during relaxation. The bond order parameter $Q_{l}$ is plotted for an example which converts from usp with $7.5 \%$ defects to fcc. The arrows indicate the values of a perfect usp-decoration (suffix i) and a perfect fcc-lattice (suffix f). $Q_{2}$ and $Q_{10}$ are zero in both structures, $Q_{4}, Q_{8}$, and $Q_{14}$ are zero for icosahedral bond order the density of the fcc-structure is higher than of the uspdecoration, but the volume of the simulation box is kept fixed during relaxation, holes are generated for optimization of nearest neighbour distances at low energy cost. Although it is known that a volume compression during relaxation can improve the final state we have not applied this method up to now because we would have to make larger modifications of the relaxation procedure. Subtracting the binding energy of the missing atoms from $E_{b}^{\text {fcc }}$, one obtains the value of $E_{b} \approx 3.0$ of Fig. 6 .

Figure 7 presents for $n_{d}=7.5 \%$ the convergence of $Q_{l}(l=4,6,8,12,14)$ to the exact values of the fcc-lattice. Initially one observes a rapid decrease for the high harmonic $Q_{12}$ which is caused by the distortion of the quasilattice close to the defects. The decrease slows down until a sudden transition takes place to the fcc-values, indicat- ing a large scale reordering of the atoms. The bond order parameters of lower degree rise continuously from the initial value which indicates that the phase transition from an icosahedral to the fcc structure is also continuous. In Fig. 9 the transition is shown by projections of the bonds onto an octant. The bonds which were aligned along few directions at first are scattered in the transition state between icosahedral and crystalline but at last get aligned again along the directions of the cubic bonds.

For structures which finally become crystalline the binding energy frequently approaches the typical energy of the icosahedral phase first. In this time intervall the quasilattice is distorted to compensate the missing atoms and the hard core of the interstitials. Afterwards the reordering to the crystalline phase takes place. Depending on the initial defect configuration, the intermediate time 


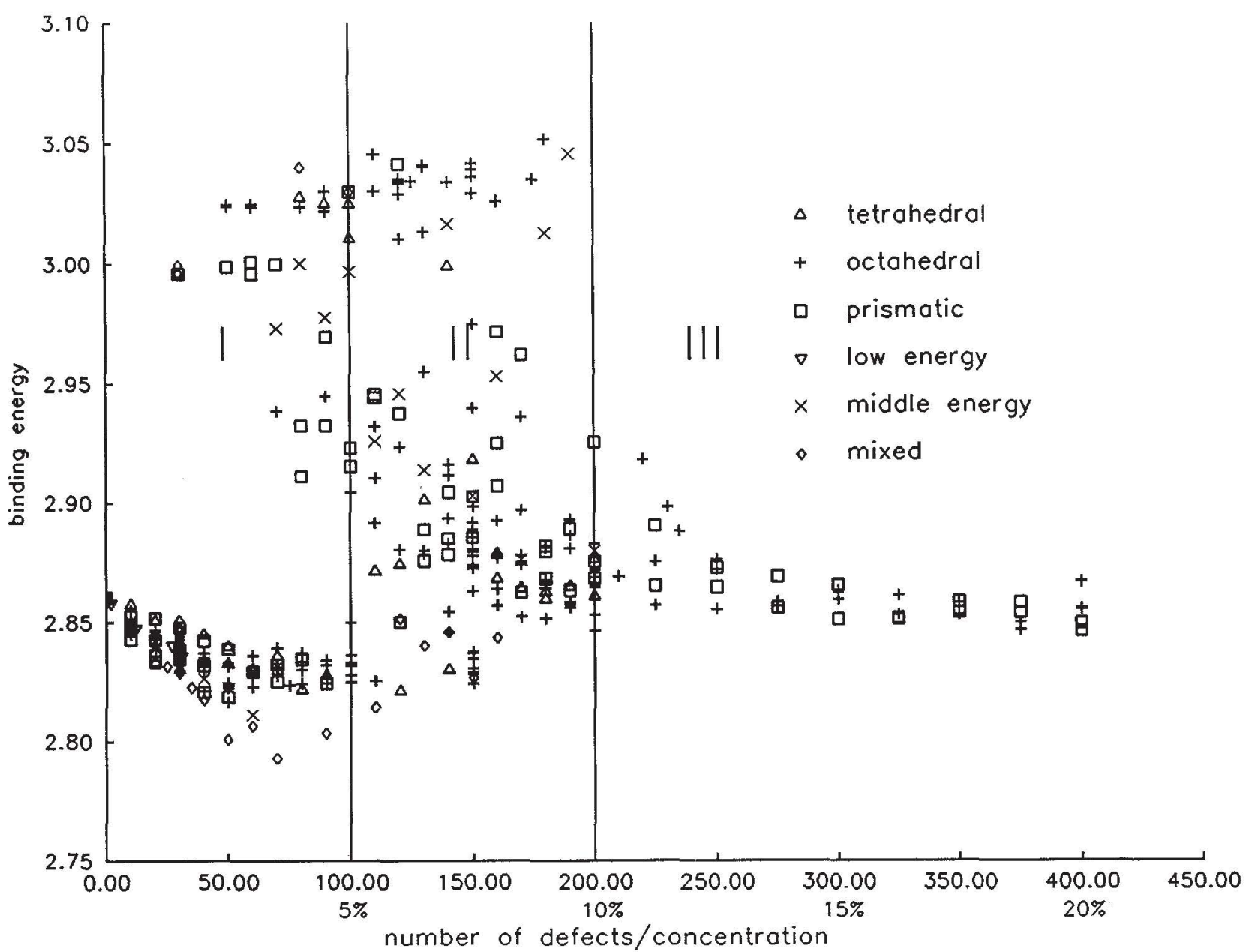

Fig. 8. Summary of the binding energies of all simulation runs. The symbols for the different types of interstitials and defects, respectively are shown in the diagram and refer to the types of defects introduced in Table 2. Mixed means that defects were generated regardless of their radius and shape. The vertical lines indicate the boundaries between the three sectors discussed in the text

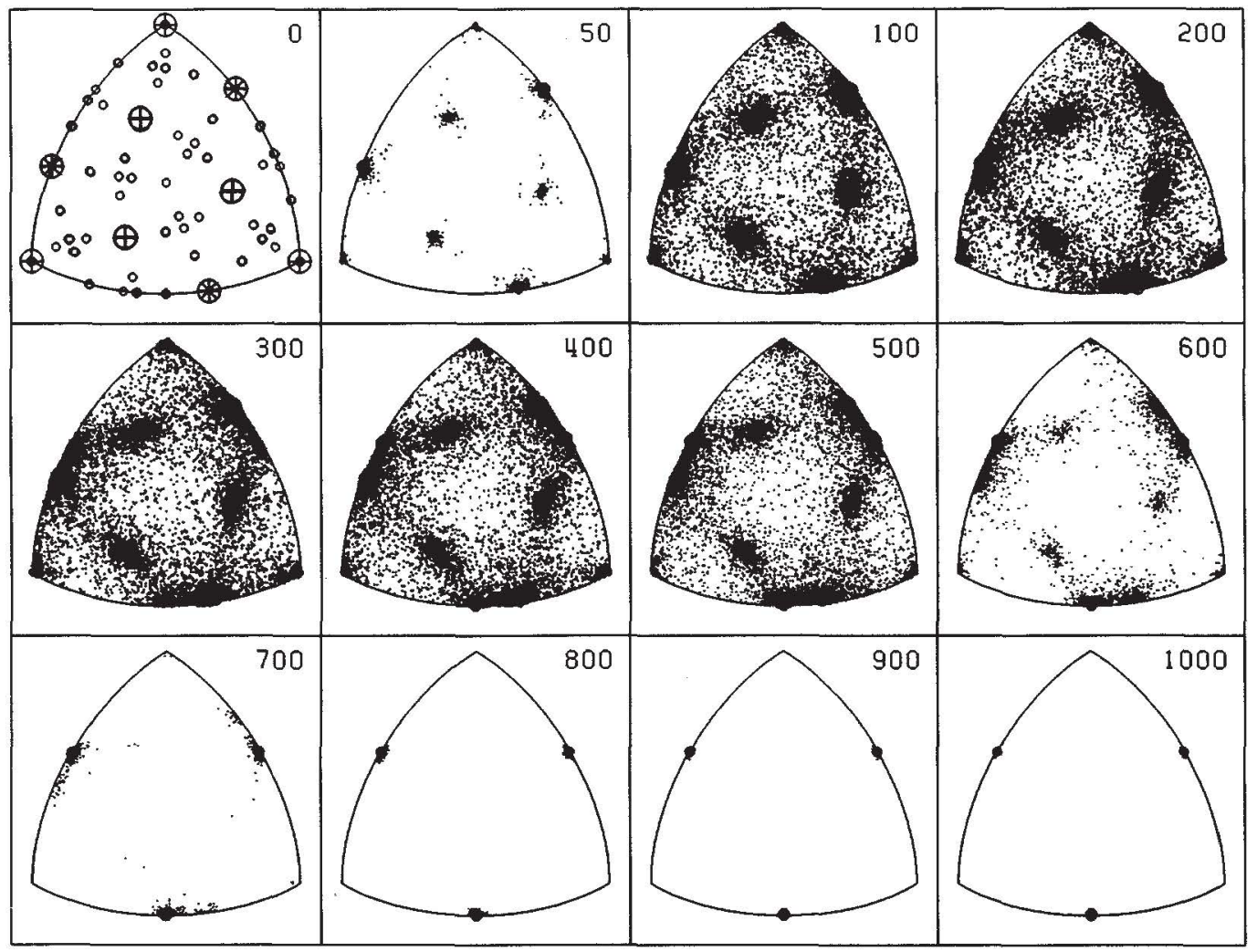

Fig. 9. The evolution of the bonds during relaxation for an example which converts from usp with $7.5 \%$ defects to fcc. An octant spanned by the pairwise orthogonal twofold symmetry axes of an icosahedron is drawn. The numbers indicate the relaxation steps. The diameter of the points is proportional to the number of bonds in this direction except for the first diagram. If the order is low the diagrams appear more densely filled with points because of the greatly varying orientations of the bonds. The encircled stars at the border of the first diagram are the five-fold bonds, and the encircled crosses are the two-fold ones. The small circles indicate the additional bonds created by the defects. The last diagram clearly shows the orientation of the bonds along a two-fold axes in a cubic structure 


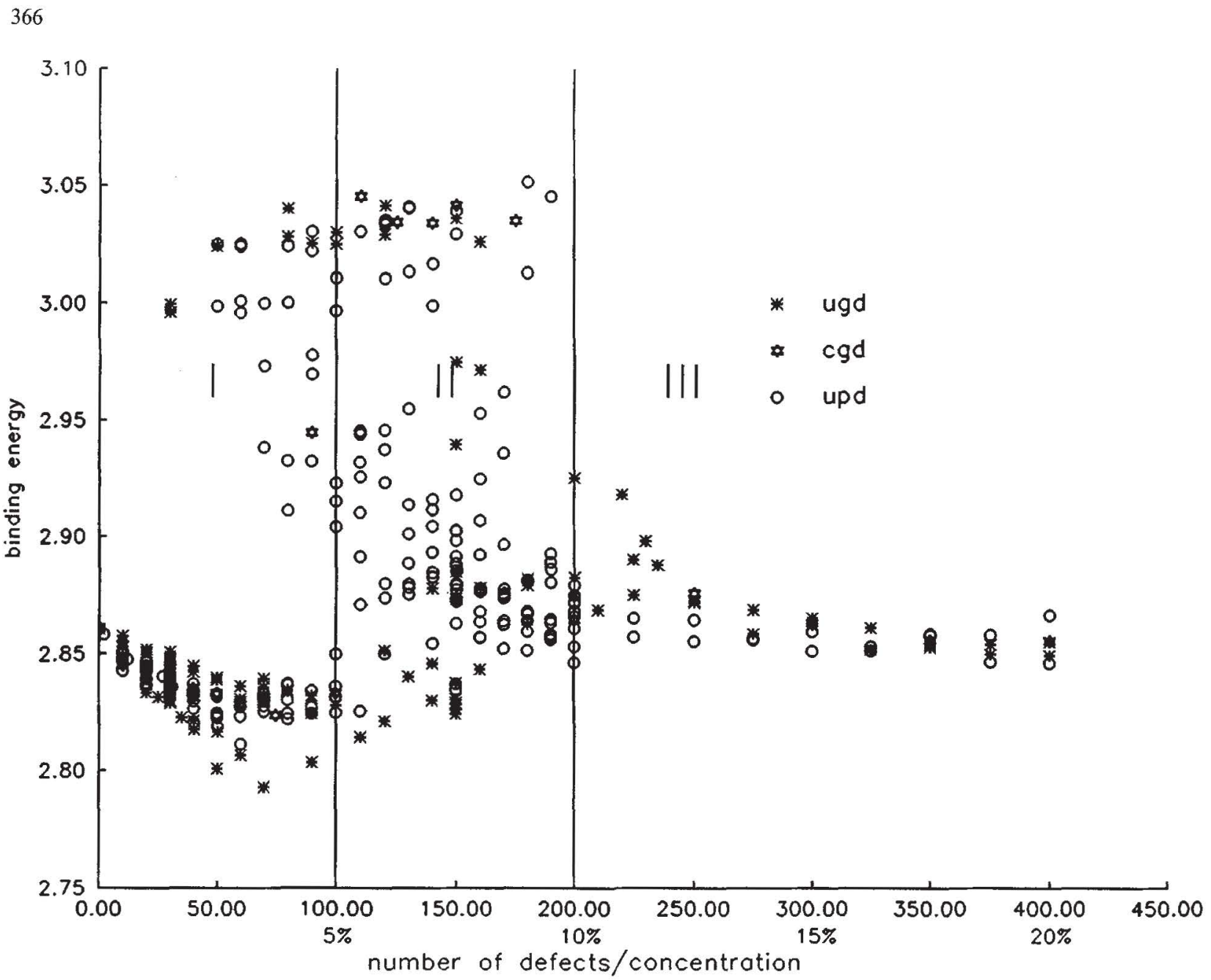

Fig. 10. The binding energies for correlated and uncorrelated geometric defects only and for physical defects, respectively. The symbols for the different types of interstitials and defects, respectively are shown in the diagram and refer to the different methods to generate the defects. Only the shorthand symbols are given. The vertical lines indicate the boundaries between the three sectors discussed in the text

interval can be rather long with a slowly increasing binding energy.

Before discussing the three characteristic sectors we want to stress that the final configuration may depend on the realization. The decomposition of $n_{d}$ into three sectors yields only majority behaviour. Whether the final states become unique for the number of atoms $N \rightarrow \infty$ remains open. 12).

We now discuss the five different structures (Figs. 8-

I. $n_{d} \lesssim 5 \%$. The inital defected icosahedral structure is (meta-)stable. No pair recombination occurs, only a lattice distortion, and therefore the binding energy (Fig. 8) decreases weakly from 2.86 to 2.78 . The rdf (Fig. 11 a) displays small, but distinct peaks at the positions of the perfect structure. The first peak is split like in the ideal usp-decoration. The bond-directions (Fig. 12a) are the same as for the perfect structure and point to the twoand five-fold axes. The bond order parameter for the lowest nontrivial non-zero degree, $Q_{6}$ (Fig. 12b), is nearly constant, whereas the reduction of $Q_{12}$ (Fig. $12 \mathrm{c}$ ) indicates that the long-range orientational order decays. $A$ low concentration of defects does not destabilize the uspdecoration.

II. $5 \% \leqq n_{d} \leqq 10 \%$. Within this range three different final structures are possible. Their occurrence does depend neither on $n_{d}$ nor on the type of defects but only on the particular realization. (Minor statistical differences in the frequency occur only between geometrical and physical defects.) The three structures are:

(a) Disordered icosahedral. The binding energy (Fig. 8) is low, between 2.82 and 2.87. The rdf (Fig. 11a) has distinct, but broad and overlapping peaks which remain at the position of the ideal usp-decoration. Therefore no reordering of the structure occurs. The bond density (Fig. 12a) has maxima along preferred icosahedral directions, but broadens. $Q_{6}$ (Fig. 12b) covers a wide range of values without systematic development whereas $Q_{12}$ (Fig. 12c) decreases rapidly. Compared to range I, here recombinations and probably minor reordering occurs and increases the binding energy. This structure preferably arises for the geometric defects, which have a higher initial energy than the physical ones. 

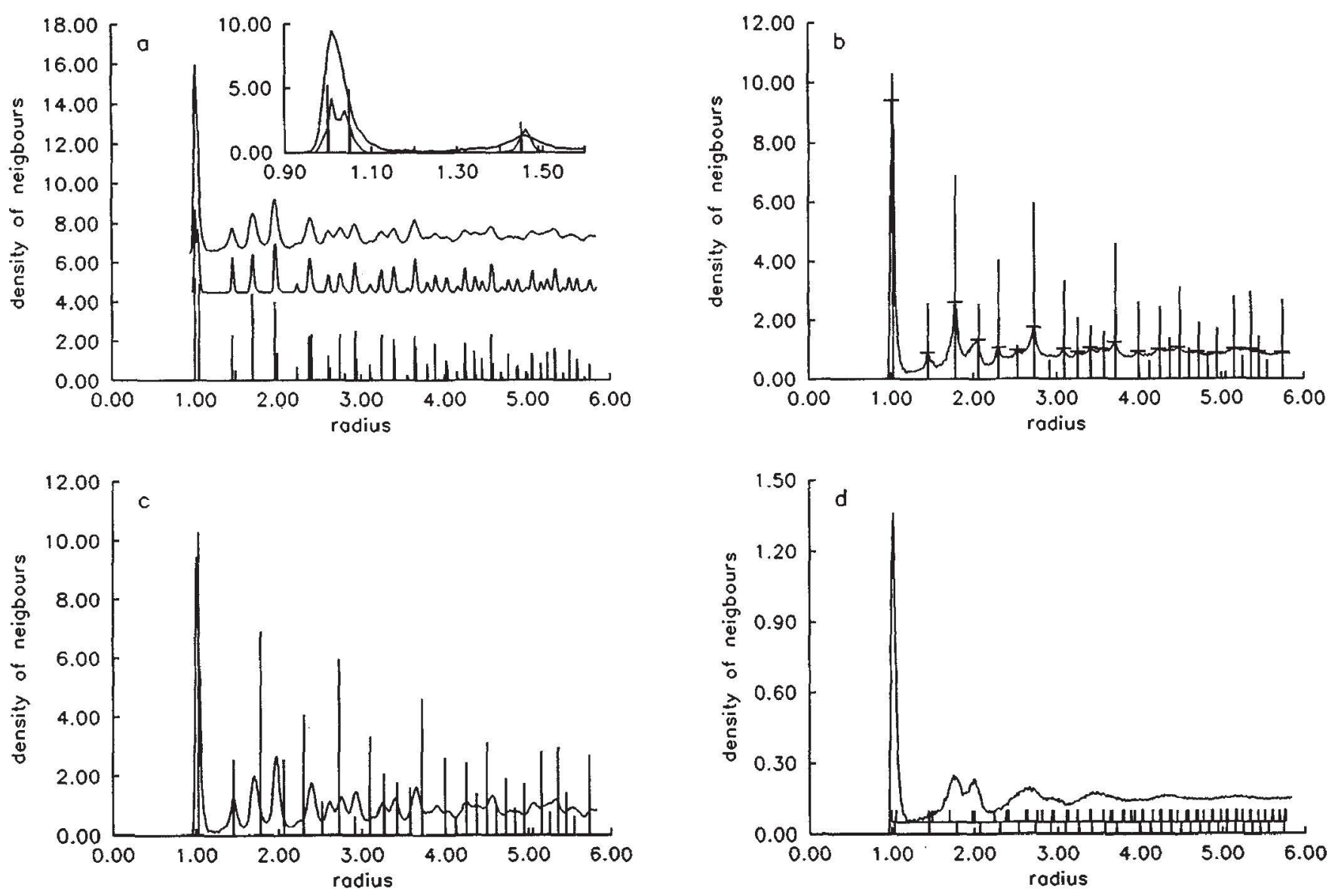

Fig. 11. The radial distribution functions: a for the icosahedral structures. The rdf of a perfect icosahedral usp-decoration (delta peaks), of the relaxed (narrow, distinct peaks), and of the disordered icosahedral structure (broad, overlapping peaks) are given. The nearest neighbour peak of the relaxed structure remains split! b for the fcc structures. The delta peaks are the rdf of a perfect fcc material, the continous function is the rdf of the disordered fcc structure. The sharp maxima of the disordered structures clearly show the remaining fcc bond ordering, but for the disordered struc-

ture especially the third, the fourth and the sixth maximum also coincide well with the shells in the amorphous structure; $\mathrm{c}$ for the ideal fcc and the disordered icosahedral structure. The positions of the maxima do not coincide in general; $d$ for the amorphous structure. The upper small peaks are the atomic separations occuring in the perfect unrelaxed icosahedral structure and the lower ones in a fec structure. The second peak of the fec and icosahedral structure is missing in the amorphous case!

(b) Disordered fcc. The binding energy (Figs. 8, 10) is widely spread from the value 2.85 of the icosahedral to 2.97 of the perfect fcc structure. In the rdf (Fig. 11b) two features are striking: sharp maxima of the continuous distribution coincide with the positions of the perfect monocrystalline fce structure, but the general behaviour of the rdf is similar to the amorphous structure (Fig. 11d) of range III. The maxima of the broad bond density (Fig. 12a) point to the directions of the ideal fcc structure. $Q_{6}$ and $Q_{12}$ (Fig. $12 \mathrm{~b}, \mathrm{c}$ ) are smeared out between the values of the icosahedral and ideal fcc structure.

Rdf and bond diagrams show, that the structure is filled with imperfect fcc-domains which are mutually aligned. Nucleation of the domains has started at many points simultaneously and gives rise to a polycrystalline state, preferably in the scheme of uncorrelated physical defects (Fig. 10).

(c) Perfect fcc. The binding energy (Figs. 8, 10) is high (2.97-3.06) and varies only because of differences in lattice constants and hole numbers during relaxation. The structure functions and diagrams (Figs. 11b, 12a c) display an almost perfect cubic structure. The coordination number (found by integrating over the first maximum of $G(r))$ is less than twelve due to the holes. The fcc structure is favoured for the high energetic geometric defects (Fig. 10).

A comparison of the $\operatorname{rdf}$ (Fig. $11 \mathrm{c}$ ) with the disordered icosahedral structure indicates that now the translational as well as the orientational order (Fig. 12a) is changed totally.

III. $n_{d} \gtrsim 10 \%$. The binding energy (Fig. 8) decreases from 2.93 to 2.85 . The rdf (Fig. 11d) is typical for an amorphous material, the bond diagram (Fig. 12a) is isotropic, $Q_{6}$ (Fig. 12b) and $Q_{12}$ (Fig. 12c) are small. 


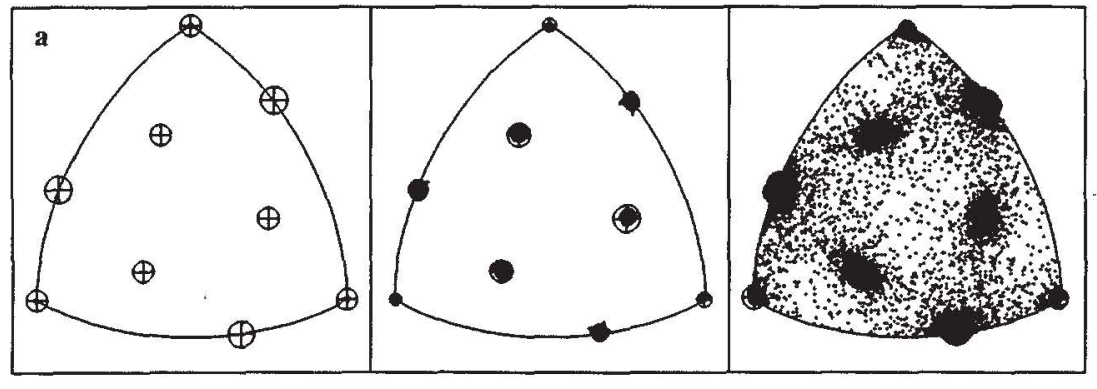

perf.icos. rel.icos.

dis. Icos.

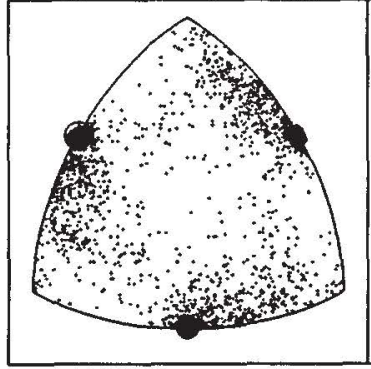

dis.fcc

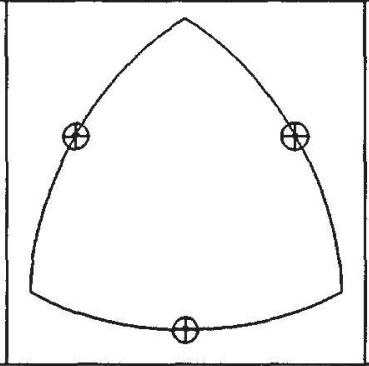

perf.fcc

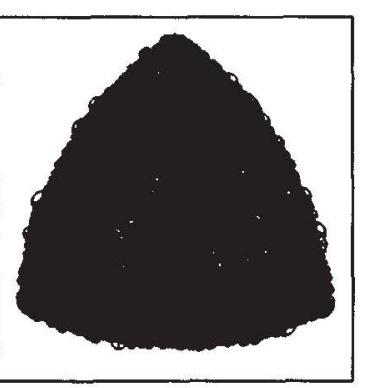

omorphous
Fig. 12. The bond orientational order: a The bond diagrams for the ideal icosahedral and the five final structures. For explanation see also Fig. 9. The short hand notations stand for perfect icosahedral, relaxed icosahedral, disordered icosahedral, disordered fcc, and perfect fcc; b The bond order parameter $Q_{6}$. Its value for the ideal icosahedral bond order is 0.187 , for cubic face-centered it is 0.575 ; $\mathbf{c}$ The bond order parameter $Q_{12}$. Its value for the ideal icosahedral bond order is 0.498 , for cubic face-centered it is 0.600
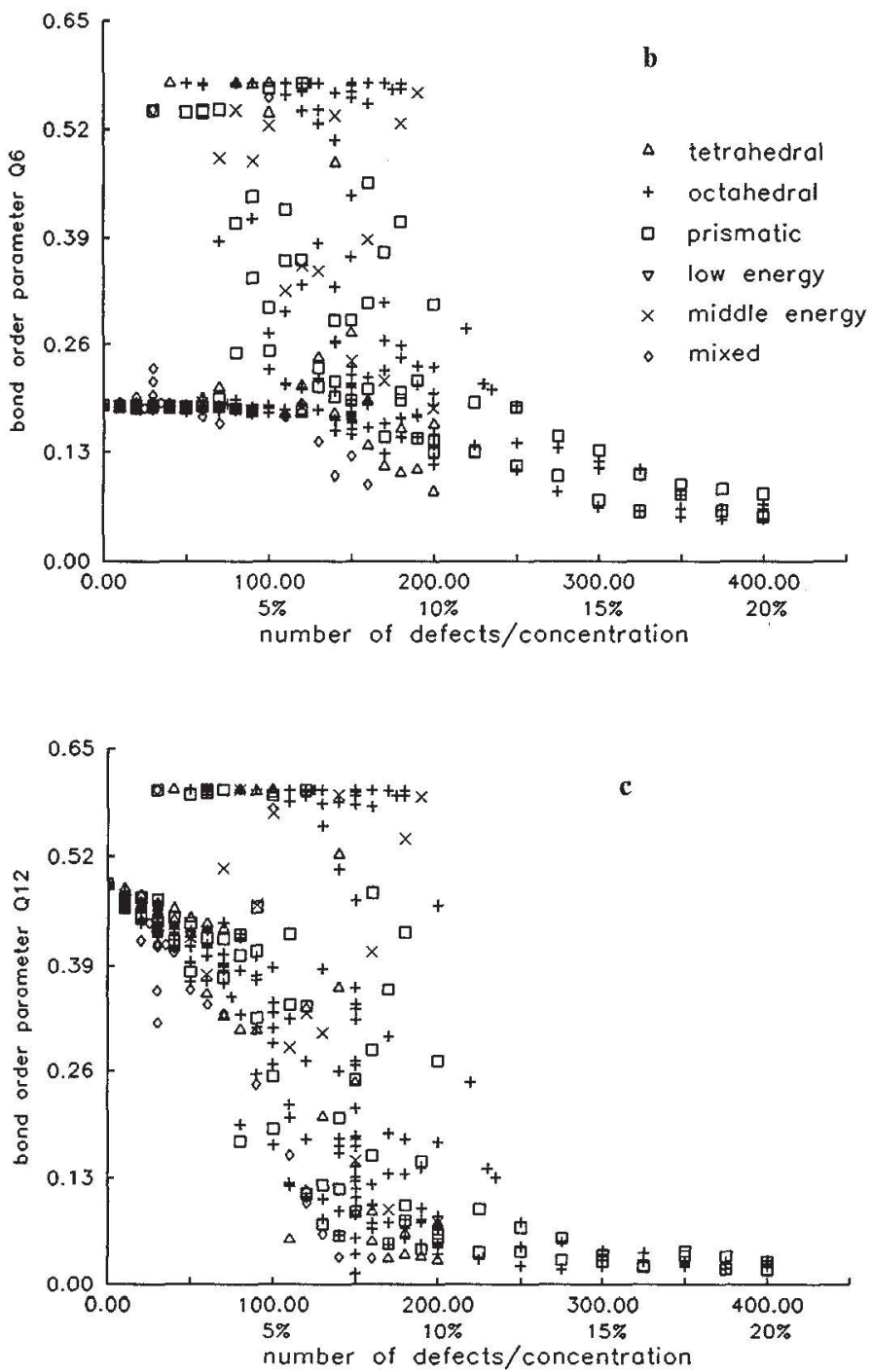

\section{Summary and discussion}

The purpose of the present paper has been an extension of our previous studies on the stability of quasicrystals. In particular we have investigated the stability under the generation of Frenkel defects.

In a first step we have classified the interstitial sites for a quasicrystal with usp-decoration geometrically and energetically. This decoration has been found metastable (at $T=0 \mathrm{~K}$ ) in our recent work [1]. There are 16 different geometrical interstitial sites out of which only 8 are stable.

The binding energy was used to characterize the stability, the rdf to describe the translational order, and bond diagrams as well as the orientational order parameter to display the orientational order.

With respect to the nature of the relaxed states, sectors for the concentration $n_{d}$ of the Frenkel pairs could be identified. For $n_{d} \geqq 5 \%$ the initial disorder is not large enough to destroy the overall icosahedral order. Between about $5 \%$ and $10 \%$ there is a competition between icosahedral and fcc ordering, which is related to the following facts:

The nearest neighbour bonds of the usp-decoration point to the five-fold and two-fold axes (Fig. 9, first picture, $\oplus$ and $\otimes$ ). If there are octahedral defects in the sample, further bonds occur along the three-fold axes, in mirror planes and in general directions (Fig. 9, first picture, o). During the relaxation the bonds from fivefold directions move to the nearby mirror planes. All others disappear step by step. What remains are the nearest neighbour connections of a fcc-lattice (Fig. 9, last picture) along the twofold axes of the cubic symmetry. Only minor local changes are necessary to transform the structure form icosahedral to crystalline. 
For $n_{d}$ larger than about $10 \%$ the generated disorder is large enough to hinder a relaxation to an icosahedral or fcc structure. The final structure is typically an amorphous one.

Let us now return to the experimental data of Urban et al. $[3,4]$. A quantitative comparison with their results is not possible because we have studied only a monatomic quasicrystal with simple structure. Atoms can move to a local minimum close to their initial position. If the number of defects is low enough they also may recombine. Diffusion is not possible because of lacking kinetic energy and potential barriers. Our results are therefore only valid near $T=0 \mathrm{~K}$. A finite temperature irradiation and a higher temperature annealing require a full-scale molecular dynamics or Monte Carlo simulation.

But nevertheless our results demonstrate that for $n_{d} \gtrsim 10 \%$ the icosahedral quasicrystal with usp-decoration becomes unstable and finally relaxes to an amorphous configuration which is in qualitative agreement with the experiment. Of course the annealing from the amorphous to the quasicrystalline structure cannot be investigated by our method.

In contrast to the theory of Kronmüller et al. [6], which presents critical defect concentrations of $0.2 \%[6]$ and $2.0 \%[7]$, we find stability of the icosahedral phase up to $10 \%$ and an intermediate range of relaxation to a crystalline state. This theory, however, is not specific to quasicrystals.

The case of monatomic quasicrystals with Frenkel defects is very restrictive as no stable monatomic quasicrystals have been observed in nature to date. In addition Frenkel defects, too, are eliminated in monatomic materials because of selfdiffusion. Nevertheless our studies yield information about single vacancies and single interstitials in quasicrystals. We have constructed both vacancies and interstitials randomly (ugd and upd scheme), and therefore they are independent at low density. Our results claim that they do not destabilize the icosahedral structure.

The tools developed for the monatomic case have been modified for diatomic quasicrystals. We have studied a simplified model of the $\mathrm{Al}-\mathrm{Li}-\mathrm{Cu}$-structure containing only tetrahedral interstitials. The results will be published in a forthcoming paper.

\section{References}

1. Roth, J., Schilling, R., Trebin, H.-R.: Phys. Rev. B41, 2774 (1990)

2. Lançon, F., Billard, L.: J. Non-Cryst. Solidi 117/118, 836 (1990)

3. Urban, K., Moser, N., Kronmüller, H.: Phys. Status Solids A91, 411 (1985)

4. Urban, K., Mayer, J., Rapp, M., Wilkens, M., Csanady, A., Fidler, J.: J. Phys. (Paris) 47, C3-465 (1986)

5. Bauer, M.: Diploma Thesis, Stuttgart 1987

6. Kronmüller, H.: Cryst. Lattice Defects Amorph. Matter 14, 137 (1987)

7. Kronmüller, H., Hoffmann, A., Bauer, M.: In: Quasicrystalline materials. Proc. of the I.L.L./CODEST Workshop in Grenoble. Janot, Ch., Dubois, J.M. (eds.), p. 29. Singapore: World Scientific 1988

8. Steinhardt, P.J., Nelson, D.R., Ronchetti, M.: Phys. Rev. B28, 784 (1983)

9. Zallen, R.: The physics of amorphous solids. New York: Wiley 1983

10. Henley, C.L.: Phys. Rev. B34, 797 (1986)

11. Lançon, F., Billard, L.: J. Phys. (Paris) 51, 1099 (1990)

12. Olami, Z., Alexander, S.: Phys. Rev. B37, 3973 (1988) 